Western University Scholarship@Western

Summer 2017

\title{
Effects of Protein Deficiency on Perinatal and Postnatal Health Outcomes
}

Shelby L. Oke

Western University, soke2@uwo.ca

Daniel B. Hardy

Physiology and Pharmacology, daniel.hardy@schulich.uwo.ca

Follow this and additional works at: https://ir.lib.uwo.ca/physpharmpub

Part of the Medical Physiology Commons, Pharmacy and Pharmaceutical Sciences Commons, and the Reproductive and Urinary Physiology Commons

Citation of this paper:

Oke, Shelby L. and Hardy, Daniel B., "Effects of Protein Deficiency on Perinatal and Postnatal Health Outcomes" (2017). Physiology and Pharmacology Publications. 101.

https://ir.lib.uwo.ca/physpharmpub/101 


\title{
Effects of Protein Deficiency on Perinatal and Postnatal Health Outcomes
}

\author{
Shelby L. Oke and Daniel B. Hardy
}

\begin{abstract}
There are a variety of environmental insults that can occur during pregnancy which cause low birth weight and poor fetal health outcomes. One such insult is maternal malnutrition, which can be further narrowed down to a low protein diet during gestation. Studies show that perinatal protein deficiencies can impair proper organ growth and development, leading to long-term metabolic dysfunction. Understanding the molecular mechanisms that underlie how this deficiency leads to adverse developmental outcomes is essential for establishing better therapeutic strategies that may alleviate or prevent diseases in later life. This chapter reviews how perinatal protein restriction in humans and animals leads to metabolic disease, and it identifies the mechanisms that have been elucidated, to date. These include alterations in transcriptional and epigenetic mechanisms, as well as indirect means such as endoplasmic reticulum (ER) stress and oxidative stress. Furthermore, nutritional and pharmaceutical interventions are highlighted to illustrate that the plasticity of the underdeveloped organs during perinatal life can be exploited to prevent onset of long-term metabolic disease.
\end{abstract}

\section{Keywords}

DOHaD - Amino acids - Liver - Adipose - Pancreas - Maternal LP diet • Diabetes $\cdot$ Dyslipidemia $\cdot$ Longevity $\cdot$ Epigenetics $\cdot$ Posttranslational histone

S.L. Oke

The Departments of Obstetrics and Gynecology and Physiology and Pharmacology, The University of Western Ontario, London, ON, Canada

e-mail: soke2@uwo.ca

D.B. Hardy $(\bowtie)$

The Departments of Obstetrics and Gynecology and Physiology and Pharmacology, The Children's Health Research Institute and the Lawson Health Research Institute, The University of Western

Ontario, London, ON, Canada

e-mail: Daniel.Hardy@schulich.uwo.ca 
modifications $\bullet$ DNA methylation $\bullet$ Endoplasmic reticulum stress $\bullet$ MicroRNAs $\bullet$ Taurine $\bullet$ Oxidative stress

\section{List of Abbreviations}

$11 \beta$-HSD1 $11 \beta$-Hydroxysteroid dehydrogenase type I

ADP Adenosine diphosphate

Akt1 Protein kinase B

ALS Amyotrophic lateral sclerosis

Cyp1A2 Cytochrome P450 1A2

Cyp2c11 Cytochrome P450 2c11

Cyp3a1 Cytochrome P450 3al

Cyp7a1 Cholesterol 7 alpha-hydroxylase

DOHaD Developmental origins of health and disease

ER stress Endoplasmic reticulum stress

G6Pase Glucose-6-phosphatase

GLUT4 Glucose transporter type 4

GR Glucocorticoid receptor

GRP78 Glucose-regulated protein 78

IGF-1 Insulin growth factor 1

IRS-1 Insulin receptor substrate 1

IUGR Intrauterine growth restriction

LDL Low-density lipoprotein

LP Low protein

LPL Lipoprotein lipase

LXR Liver $X$ receptor

LXRE LXR response element

MEF2 Myocyte enhancer factor-2

miRs MicroRNAs

MPR Maternal protein restriction

p-eIF2 $\alpha$ Phosphorylated eukaryotic translation initiation factor 2

PND Postnatal day

PPAR $\alpha$ Peroxisome proliferator-activated receptor alpha

PPAR- $\gamma$ Peroxisome proliferator-activated receptor gamma

ROS Reactive oxygen species

SAM Severe acute malnutrition

SGA Small for gestational age

SIRT1 Sirtuin 1

UPR Unfolded protein response

XBP1 X-box binding protein 1

\section{Contents}

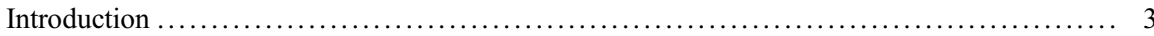

Protein Restriction and Long-Term Outcomes: Clinical Evidence ..................... 4

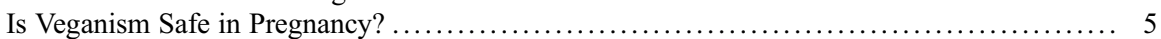

Maternal Protein Restriction (MPR) Rodent Model: Relevance to Human IUGR ............ 6 


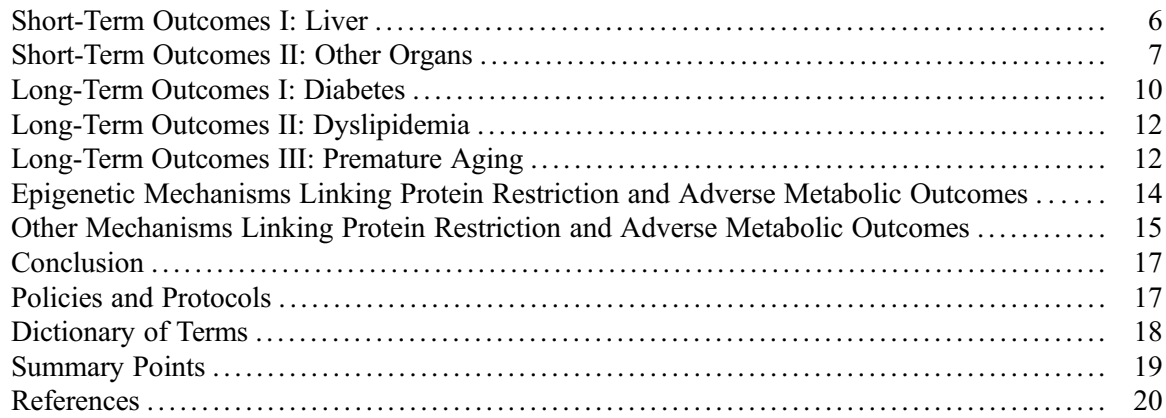

\section{Introduction}

There are a variety of insults that can occur during pregnancy leading to intrauterine growth restriction (IUGR). IUGR is characterized by a delay in fetal growth rate; therefore, IUGR infants are often categorized as being small for gestational age (SGA) due to low birth weight. One of the most common insults that can prompt IUGR is maternal malnutrition, a global problem across all classes of socioeconomic status. Over the past half century, a sizable amount of evidence has revealed the important relationship between birth weight and postpartum development (Barker 1994; Ong et al. 2000). One of the leading contributors to this finding was Dr. David Barker, an English epidemiologist who is well known for establishing the "Predictive Adaptive Response" hypothesis (Hales and Barker 2001). This hypothesis is highly supportive of the developmental origins of health and disease (DOHaD), as it suggests that unfavorable in utero events can permanently alter physiological processes that lead to the metabolic syndrome. The hypothesis states that fetal programming is altered in preparation of a nutritionally scarce postnatal environment, thereby producing a "thrifty" phenotype that is characterized by fetal energy conservation (Hales and Barker 2001). Unfortunately, these metabolic adaptations become harmful when the fetus is born into a nutritionally rich environment because the neonate is programmed to store energy rather than spend it. Individuals who are affected by this thrifty phenotype therefore tend to become obese early in life and have an increased risk for early-onset type II diabetes mellitus, cardiovascular disease, and stroke among other chronic conditions (Ravelli et al. 1998; Eriksson 2006; Barker et al. 2002).

The composition of maternal diet during pregnancy plays a large part in fetal development, as an absence or excess of nutrients can impact organ growth and development. Maternal malnutrition can exist in a variety of forms, including global nutrient abnormalities (i.e., high or low caloric intake) or atypical supplementation of specific macromolecules and nutrients. Regardless of the source, human and animal studies have demonstrated that maternal malnutrition in pregnancy also leads to placental insufficiency, an idiopathic condition by which reduced maternofetal nutrient transfer leads to IUGR (Ogata et al. 1986; Simmons et al. 1992). One such model is the maternal protein restriction (MPR) model of undernutrition, which investigates the impact of perinatal protein deficiency in IUGR offspring. Amino acids have been 
shown to be critical for fetal growth and development, as they are the structural building blocks for all proteins (Crosby 1991; Petry et al. 2001). Inadequate supplementation of amino acids during pregnancy has been shown to cause asymmetrical IUGR, as LP animal offspring have reduced growth of organs such as the liver, muscle, and pancreas at the expense of more essential organs like the brain (Desai and Hales 1997). These offspring consequently have impaired metabolic programming that persists into adulthood, and thus exhibit a phenotype that is characteristic of the metabolic syndrome. Moreover, as Barker's hypothesis would suggest, MPR offspring that are fed a normal protein diet after birth undergo rapid growth during early periods of life (Ozanne and Hales 2004). Moreover, postnatal catch-up growth exacerbates the symptoms and incidence of metabolic deficits (Sohi et al. 2011; Bol et al. 2009; Bieswal et al. 2006), and this dietary mismatch also appears to have significant effects on lifespan (Ozanne and Hales 2004). Considering these changes to metabolism and longevity, this review aims to show the importance of maternal protein during pregnancy on long-term outcomes of the offspring, with an emphasis on how postnatal catch-up growth can modify the mechanisms responsible for regulation of glucose, lipids, hormones, and lifespan.

\section{Protein Restriction and Long-Term Outcomes: Clinical Evidence}

In 1986, Barker and his colleagues discovered birth records for over 15,000 English persons born prior to 1931. These records were collected by Miss Ethel Burnside, Lady Inspector of Midwives for Herfordshire, England, who documented birth weight and body weight at 1 year of age (Barker 2003). These follow-up records allowed Barker to assess the growth trajectory of individuals within the first year of life, and he was able to further inquire about adult health for those still living at the time. The data revealed that those who were born of low birth weight had disproportionately higher rates of coronary heart disease (Barker 2003; Ravelli et al. 1976), and these individuals also had impaired liver size and/or function at birth (Barker et al. 1993). This is not surprising, as IUGR often results in asymmetric organ development (Desai and Hales 1997). Furthermore, studies of individuals born around the time of the Dutch Hunger Winter reveal that prenatal exposure to famine confers increased risk for glucose intolerance in adulthood (Ravelli et al. 1998). This population also had high rates of obesity after exposure to famine during the first half of gestation (Ravelli et al. 1976), suggesting that timing of maternal malnutrition during pregnancy can influence long-term metabolic outcomes of offspring.

While the previously mentioned epidemiological studies are focused on caloric restriction, there is also evidence to support that protein deficiency during critical periods of development gives rise to poor metabolic outcomes. Populations of children with severe acute malnutrition (SAM) are often used to study the repercussions of malnutrition, as these individuals see the effects of a low calorie diet (marasmus) or a low protein, high carbohydrate diet (kwashiorkor; Forrester et al. 2012; Spoelstra et al. 2012). In 1967, a study of Ugandan children revealed that individuals with kwashiorkor had low serum protein levels in comparison to those 
with marasmus (Hadden 1967). These individuals also exhibited glucose intolerance and elevated plasma free fatty acids (Hadden 1967); however, children with kwashiorkor displayed normal glucose tolerance after a 2 week dietary intervention (Hadden 1967). It was proposed that the original impairment in glucose tolerance may be due to an inability to utilize free fatty acids as a substrate in the citric acid cycle, so adequate dietary protein may be essential for normal aerobic metabolism (Hadden 1967). More recent studies also show that children with kwashiorkor exhibit reduced lipolysis and fatty acid oxidation relative to children with marasmus (Badaloo et al. 2006), while children with kwashiorkor or marasmus have pancreatic beta cell dysfunction that contributes to glucose intolerance (Spoelstra et al. 2012). Studies have also established that SAM has early life origins, as low birth weight infants have high risk for exhibiting either marasmus or kwashiorkor when exposed to a nutrient-poor postnatal environment (Francis-Emmanuel et al. 2014). Interestingly, individuals who exhibit marasmus tend to be of lower birth weight than those who develop kwashiorkor; however, individuals from both groups of SAM tend to have poor metabolic outcomes as adults (Francis-Emmanuel et al. 2014). As mentioned previously, nutrition-induced accelerated growth influences the onset of metabolic disease in low birth weight offspring (Eriksson 2006). Unfortunately, none of the discussed human SAM studies contained data on childhood growth rate, so it remains unknown as to whether catch-up growth is involved in metabolic outcomes of individuals who experienced SAM in early life. Furthermore, because a typical kwashiorkor diet has low protein and high carbohydrate content, it is not clear whether long-term metabolic dysfunction occurs in adulthood due to low dietary protein, high carbohydrates, or both for these individuals.

\section{Is Veganism Safe in Pregnancy?}

Veganism and vegetarianism is also of interest when studying the effects of protein restriction, as a vegan/vegetarian diet relies solely on plant-sourced nutrients. Individuals who practice veganism or vegetarianism must be careful to ensure that they ingest an adequate amount of protein, often in the form of legumes, lentils, grains, etc. There are mixed opinions on whether consumption of a vegan/vegetarian diet is safe during pregnancy, as observational human studies report conflicting data on both maternal and fetal outcomes. A literature review by Piccoli et al. (2015) revealed that multiple studies found infants of vegetarian mothers to be of lower birth weight than nonvegetarian mothers, while two different studies reported that infants of vegans/vegetarians actually have higher birth weight and length. Gestational age was not disclosed for either of these studies; therefore, the association between a vegetarian/vegan diet and high birth weight is not necessarily meaningful (Piccoli et al. 2015). It was also noted that most studies did not report maternal protein intake levels, so it is hard to conclude whether there is a relationship between veganism/vegetarianism and fetal outcomes. Moreover, a case report by Mariani et al. (2009) revealed poor short-term outcomes of an infant born to a vegan mother. The infant had been breast-fed exclusively up until 10 months of age and showed 
developmental delay, failure to thrive, and megaloblastic anemia among other conditions (Mariani et al. 2009). Furthermore, the infant exhibited major improvement with vitamin supplementation, so it may be that the health impairments were due to vitamin deficiencies rather than low protein (Mariani et al. 2009). Regardless of these reports, organizations such as the American Dietetic Association maintain that a vegan or vegetarian diet is safe during pregnancy (Craig and Mangels 2009). That said, physicians must assess protein intake of pregnant women who consume these diets, and future studies are warranted to determine any long-term detrimental effects on offspring.

\section{Maternal Protein Restriction (MPR) Rodent Model: Relevance to Human IUGR}

Protein and amino acids are an essential part of the human diet, and many studies have determined that amino acids have a key role in fetal growth and development (Crosby 1991; Petrik et al. 1999). An absence of amino acids is known to occur in cases of both maternal malnutrition and placental insufficiency, thereby leading to low birth weight and asymmetrical IUGR. It is for this reason that the MPR model can be used to study fetal undernutrition in response to maternal malnutrition or placental insufficiency. With the MPR model, pregnant rat dams are fed a diet of $20 \%$ (control) or $8 \%$ protein. Offspring born to control diet-fed dams continue to have a $20 \%$ "normal" protein throughout life, while offspring born to LP dams are assigned to one of three groups: low protein 1 (LP1), low protein 2 (LP2), or low protein 3 (LP3). LP1 offspring are fed an $8 \%$ protein diet throughout life, while LP2 offspring are fed an $8 \%$ protein diet until weaning (i.e., PND 21). Alternatively, LP3 offspring are exposed to a LP environment exclusively during gestation - these pups are fed a $20 \%$ protein diet from birth through adulthood. It is also important to note that the reduction in calories in the $8 \%$ protein diet is compensated for by the addition of carbohydrates (Fig. 1). This makes each diet isocaloric with each other, thereby eliciting no maternal stress and no changes in maternal food intake or weight gain (Fig. 2). Furthermore, while there are no differences in postnatal food intake across all dietary groups of offspring, LP offspring were lower in body weight in postnatal life compared to control offspring (Fig. 2). It is also important to note that the MPR diet is not considered to be a "high carbohydrate" diet, as the slight percent increase in carbohydrates $(13 \%)$ is negligible relative to the substantial decrease in protein content (greater than $50 \%$ ).

\section{Short-Term Outcomes I: Liver}

Studies involving the MPR model have demonstrated that mammalian fetal liver development is impaired due to the low protein insult. While there is an overall reduction in birth weight of LP offspring (Fig. 2), there is also a significant decrease in fetal liver to body weight ratio (i.e., the liver is proportionally small; Sohi et al. 2011). 


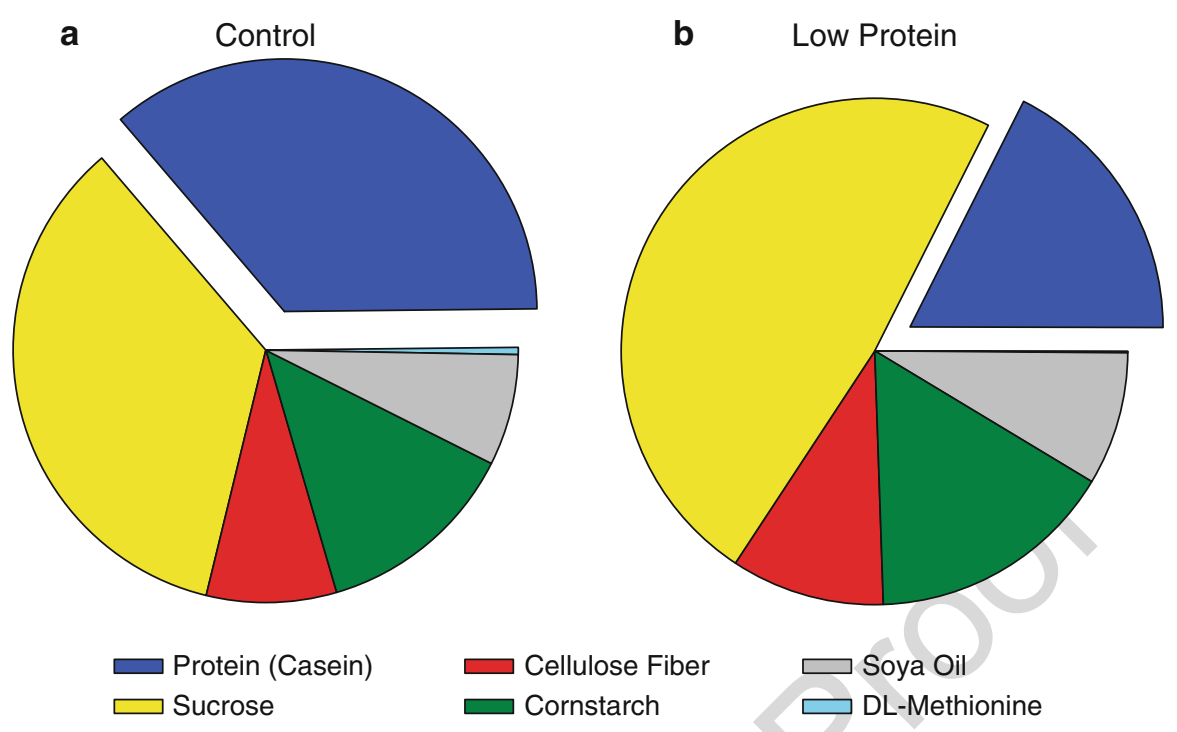

Fig. 1 Overview of control and low protein rodent diets. Composition of (a) Control $(20 \%$ protein) and (b) Low protein diet (8\% protein) are described. The low protein diet is attributed to decreased casein content but is made isocaloric by a slight increase (13\%) in carbohydrates (i.e., sucrose)

This finding suggests that fetal liver growth is compromised at the expense of more "vital" organs such as the heart and brain (Williams et al. 2005). Furthermore, the timing of protein restoration appears to be significant during the neonatal period as LP2 and LP3 offspring display liver and whole body postnatal catch-up growth despite no differences in food intake (Sohi et al. 2011). Offspring having undergone asymmetrical IUGR are believed to be prone to symptoms of the metabolic syndrome, and previous studies confirm that LP2 rat offspring exhibit glucose intolerance at PND 130 due to altered hepatic gluconeogenesis (Vo et al. 2013). In addition, adult male recuperated offspring have dyslipidemia and impaired drug metabolism due to altered expression of various hepatic cytochrome P450 enzymes (Fig. 3; Sohi et al. 2011, 2014).

\section{Short-Term Outcomes II: Other Organs}

The effects of MPR are not exclusive to the liver. Epidemiological studies indicate that there is an association between visceral obesity and poor fetal growth and this has been further confirmed via the MPR rat model (Guan et al. 2005). The increase in visceral adiposity occurs due to increased rates of preadipocyte proliferation, as indicated by increased incorporation of [3H]-thymidine into the DNA of primary rat preadipocytes (Zhang et al. 2007). It is also interesting that these studies showed no apparent alteration in preadipocyte differentiation, as there were no significant 
a
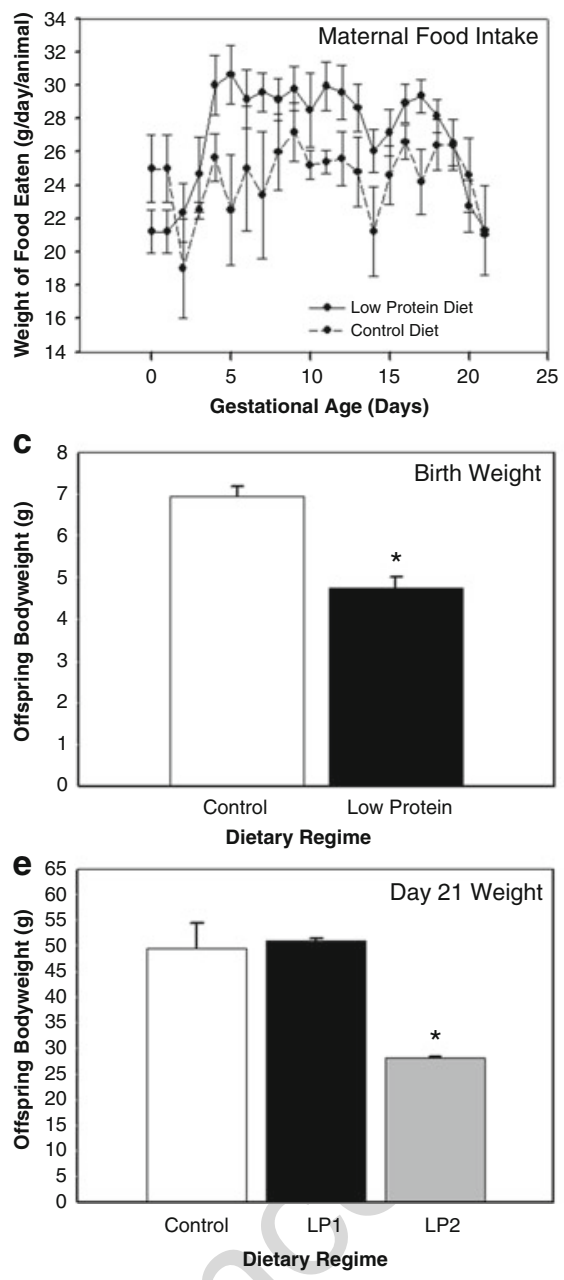

b
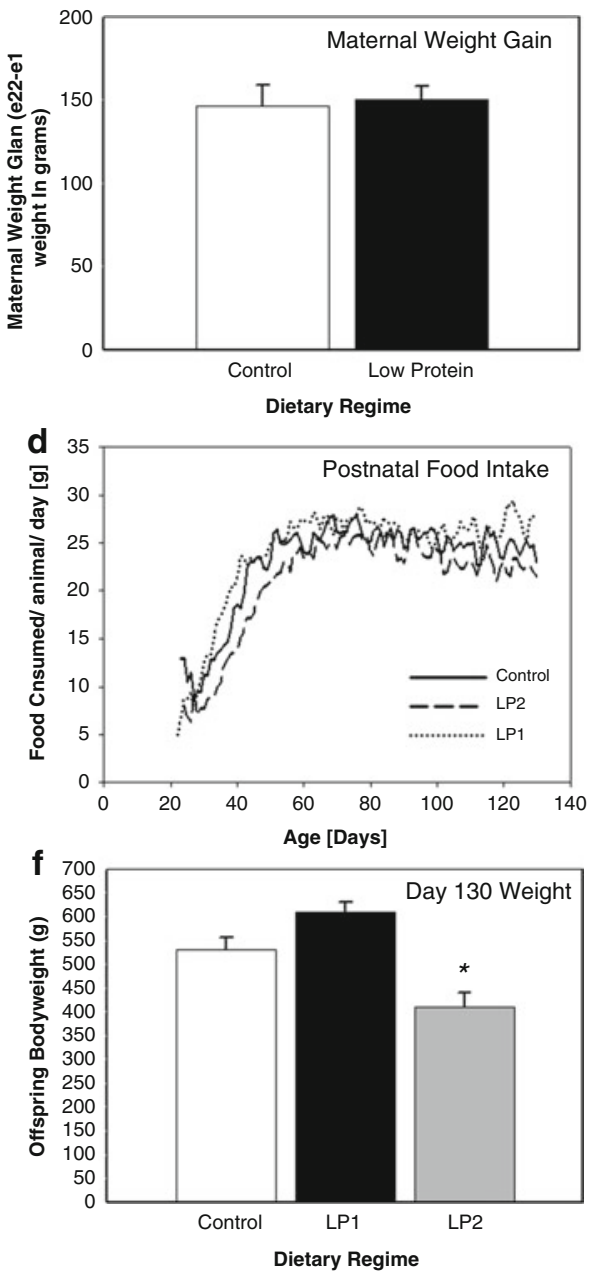

Fig. 2 Effect of maternal low protein diet on (a) Maternal food intake, (b) maternal weight, (c) birth weight, (d) food intake of offspring, (e) weight of offspring at day 21 , and (f) weight of offspring at day 130. Pregnant rats were given either a control diet (20\% protein) or a low protein diet (8\% protein) during gestation only (LP1) and lactation (LP2). Weight of food eaten in $\mathrm{g} /$ day/animal and maternal weight gain from gestation day 1 to gestation day 22 in grams were measured, respectively. Total maternal food intake, maternal weight gain, and birth weight results are expressed as the mean \pm SEM and significance was assessed using Student's unpaired t-test. For postnatal day 21 and 130 weight analysis, the dietary groups were compared by ANOVA and significant difference was determined by a Tukey HSD post hoc test for individual pairwise comparisons $\left({ }^{*} P<0.05\right.$, indicates significance between both the control and LP1 group). $n=5-8$ /group, where each $\mathrm{n}$ represents an offspring derived from a different mother (Reprinted from "Higher Hepatic MiR-29 Expression in Undernourished Male Rats During the Postnatal Period Targets the Long-term Repression of Insulin-like Growth Factor 1", G Sohi et al., Endocrinology (2015) 156(9): 3069-3076, with permission from Oxford University Press) 

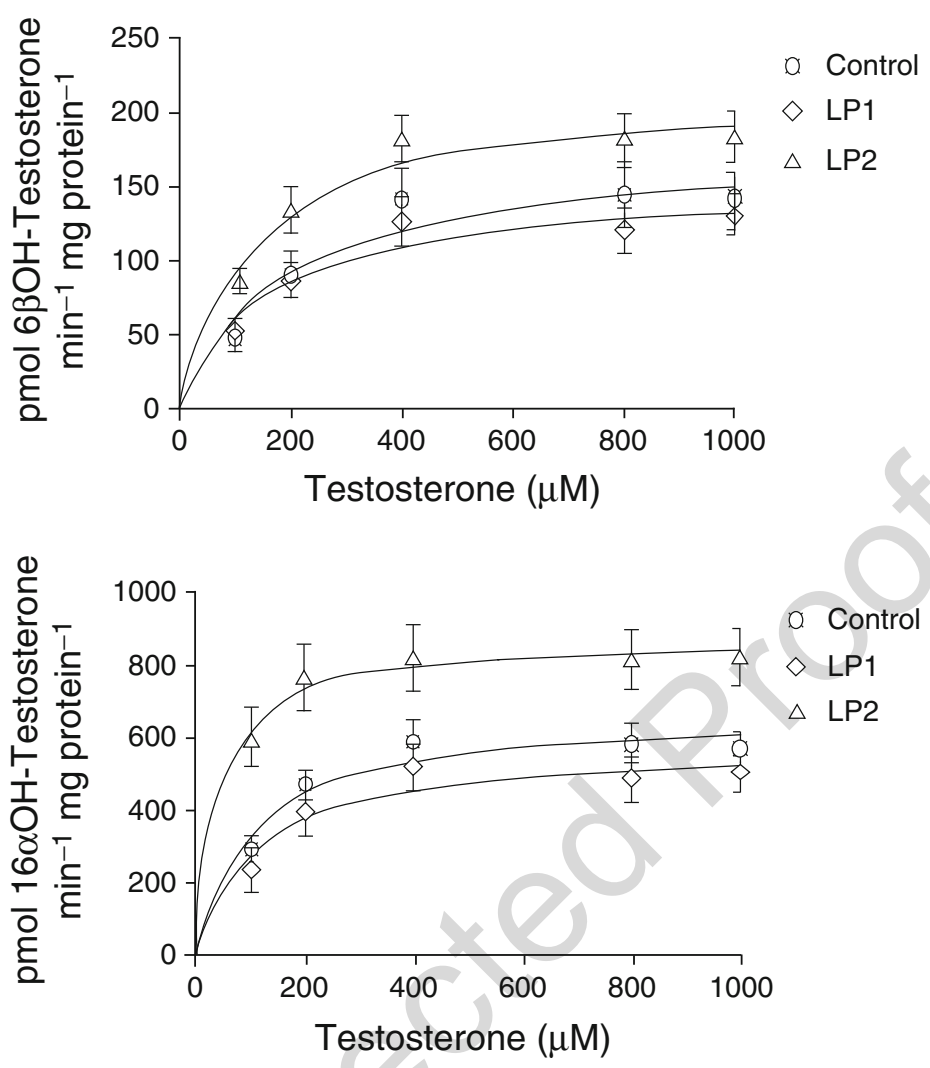

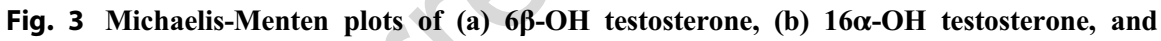
(c) $2 \alpha-\mathrm{OH}$ testosterone after incubation of day 130 rat liver microsomes (Control, LP1, and LP2) with $1 \mathrm{mM}$ NADPH and various concentrations of testosterone. Liver microsomes were extracted from control, LP1 (low protein all life), and LP2 (low protein diet during pregnancy and weaning) dietary regimes in postnatal day 130 offspring. Timed enzyme reactions were performed for testosterone metabolite analysis via solid-phase extraction followed by UPLC-PDA detection. Each data point on the curves were expressed as the mean \pm SEM. $n=5-6 /$ group, where each $n$ represents an offspring derived from a different mother (Reprinted from "Protein Restoration in Low Birth Weight Rat Offspring Derived from Maternal Low Protein Diet Leads to Elevated Hepatic Cyp3a and Cyp2c Activity in Adulthood," G Sohi et al., Drug Metabolism and Disposition (2014) 42: 221-228, with permission from The American Society for Pharmacology and Experimental Therapeutics (ASPET))

differences in the expression of peroxisome proliferator-activated receptor gamma (PPAR- $\gamma$ ) or lipoprotein lipase (LPL; Zhang et al. 2007). Early studies by Ozanne et al. (1996b) also demonstrate that MPR leads to increased insulin sensitivity of muscle at 3 months of age, as LP offspring have increased glucose uptake into skeletal muscle upon stimulation with low doses of insulin. This increased sensitivity is brought about by increased expression of GLUT4 and insulin receptors in myocyte plasma membranes (Ozanne et al. 1996a). While the mechanisms behind this are not well understood, 
it is also known that this enhanced glucose tolerance is lost later in adult life due to insulin resistance (Hales et al. 1996).

Fetal brain development also appears to be compromised by protein restriction, as LP-born rat offspring exhibit changes in kynurenine metabolism in the brain. Kynurenine metabolites are involved in neuronal development (Honório de Melo Martimiano et al. 2017), so an imbalance of these compounds within fetal brain tissue is believed to contribute to an increased risk for mental health disorders. Additionally, there is an increase in reactive oxygen species (ROS) within the brainstem of LP male offspring at weaning, so neuronal mitochondrial function may be diminished (Ferreira et al. 2016). Based on the extensive amount of studies concerned with this particular diet, it is clear that LP-born offspring have gross organ impairment contributing not only to metabolic dysfunction, but to the onset of other adult diseases as well.

\section{Long-Term Outcomes I: Diabetes}

Long-term effects to glucose homeostasis are highly promoted by maternal protein restriction, as demonstrated by glucose intolerance and insulin resistance in adult humans and adult rat offspring (Sohi et al. 2013; Chamson-Reig et al. 2009; Phipps et al. 1993). In the liver, MPR leads to hyperglycemia in 4 month offspring due augmented expression of gluconeogenic enzymes such as glucose-6-phosphatase

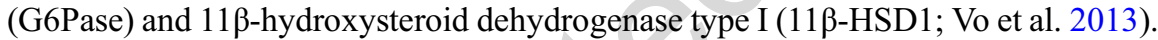
Moreover, Burns et al. (1997) demonstrated that MPR adult rats have significantly reduced hepatic glucokinase expression, thus contributing to increased glucose output. Impaired liver function leading to insulin insensitivity is further evident in MPR offspring when examining both phosphorylated eukaryotic initiation factor $2 \alpha$ (p-eIF $2 \alpha$ ) and phosphorylation of Akt1 (Sohi et al. 2013). Adult MPR offspring with postnatal catch-up growth have increased p-eIF2 $\alpha$ [Ser51], a marker of protein translation attenuation and ER stress, and this is associated with a decrease in the phosphorylation of protein kinase B (Akt1) [Ser473], a marker of insulin resistance (Sohi et al. 2013). Interestingly, MPR offspring have unchanged levels of p-eIF2 $\alpha$ at embryonic day 19; therefore, the relationship between p-eIF2 $\alpha$ and insulin sensitivity appears to be affected by postnatal catch-up growth rather than LP insult directly. This is in support of the predictive adaptive response hypothesis, as this molecular change occurs only in cases of a mismatched nutritional environment. Finally, expression of hepatic glucagon receptors was reduced fivefold in studies of MPR offspring by Ozanne et al. (1996), along with a threefold increase in hepatic insulin receptors. These changes were reflected by reduced hepatic glucose output (relative to control animals) upon stimulation with glucagon, as well as increased glucose output with administration of insulin (Ozanne et al. 1996). These studies clearly verify the importance of perinatal protein supplementation in fetal liver development, as the augmentation of many hepatic targets can negatively impact plasma glucose and insulin sensitivity.

In addition to poor outcomes seen in the developing liver, MPR appears to impact growth and function of other organs involved in glucose homeostasis, such as the 
pancreas. Epidemiological studies of adults who suffered from SAM during childhood have demonstrated that these individuals have glucose intolerance and poor insulin sensitivity later in life as a result of compromised beta cell development (Francis-Emmanuel et al. 2014). Similarly, the Preston and Hertfordshire studies by Barker and his colleagues revealed that there is an inverse relationship between birth weight, plasma glucose, and insulin concentrations of individuals exposed to famine during pregnancy (Hales et al. 1991; Phipps et al. 1993). Animal studies have since confirmed that this occurs because of reduced beta cell mass, increased islet cell apoptosis, altered beta cell cycle length and reduced pancreatic islet vascularization (Petrik et al. 1999; Boujendar et al. 2003). In cases of perinatal protein restriction, this phenotype can be rescued with administration of meat-sourced amino acids (e.g., taurine) during gestation and the first weeks of neonatal life (Boujendar et al. 2002, 2003). Supplementation of a LP diet with $2.5 \%$ taurine leads to restoration of beta cell mass by PND 130 in vivo, and in vitro studies show that this is due to normalization of DNA synthesis, apoptosis, and fetal islet vasculogenesis (Boujendar et al. 2002, 2003). A study by Chamson-Reig et al. (2006) also determined that deficient beta cell development occurs in response to MPR during early, mid, and late gestation; however, males are more susceptible to this insult during late gestation and females during mid-gestation. Not only does this emphasize that there are sex-specific differences in organ development in response to MPR, but also that timing of perinatal protein deficiency plays a role in the severity of offspring outcomes.

Studies in humans and animals also support the idea that postnatal catch-up growth confers increased risk for diabetes later in life. A study of men and women in Helsinki demonstrated that individuals who developed type II diabetes mellitus in adulthood were of low birth weight but had also caught up to average weight and height by 7 years of age. Likewise, Blesson et al. (2017) showed that female rat MPR offspring have rapid catch-up growth in the first 4 weeks of life and exhibit elevated glucose at 3 months of age. Assessment of gastrocnemius muscle from these female offspring revealed that they express altered phosphorylation of molecules involved in insulin signaling, including insulin receptor substrate-1 (IRS-1), Akt-1, and glycogen synthase. This is again in support of the idea that postnatal catch-up growth is detrimental to metabolic organ function, as in utero adaptations are not conducive in a mismatched postnatal environment. In contrast with this, Zheng et al. (2012) demonstrated that female LP offspring have increased expression of Glucose Transporter Type 4 (GLUT4) mRNA and protein in skeletal muscle at PND 38. These offspring also have increased expression of myocyte enhancer factor 2A (MEF2A), a coactivator of GLUT4 transcription, and increased glycogen synthase (Zheng et al. 2012). The authors suggest that this may be an adaptive response to MPR during gestation, and it is possible that estrogen may be involved due to the apparent sex-specific differences (Zheng et al. 2012). 


\section{Long-Term Outcomes II: Dyslipidemia}

With respect to lipids, perinatal protein appears to play a role in the maintenance of healthy cholesterol levels in adult offspring. Male rats exposed to severe MPR (4\% protein) during the last third of gestation exhibit elevated LDL and reduced highdensity lipoprotein (HDL; de Oliveira et al. 2016). In addition, male rat MPR offspring with catch-up growth show increases in cholesterol due to decreased expression of Cyp7a1, the critical enzyme in cholesterol metabolism (Sohi et al. 2011). While hepatic and circulating cholesterol was increased for both males and females at PND 21, there was an increase exclusively in males at PND 130 (Sohi et al. 2011). It is noteworthy that these adult offspring exclusively with catch-up growth (e.g., LP2 offspring) also have increased expression and activity of hepatic Cyp3a1 and Cyp2c11, which are involved in the catabolism of many drugs, including statins (Fig. 3, Sohi et al. 2014). Therefore, it is very conceivable that these animals that exhibit hypercholesterolemia also do not respond as well to cholesterolcontrolling drugs. In addition, considering that testosterone is a major substrate for these particular Cyp enzymes, this may explain why MPR male offspring have lower circulating testosterone levels, and consequentially, the long-term sexual dimorphism that exists in this model (Chamson-Reig et al. 2009).

Besides the changes seen in expression and function of hepatic Cyp enzymes, MPR also influences cholesterol levels by way of altered insulin growth factor-1 (IGF-1). IGF-1 is a hormone that is known to play a large role in fetal and placental growth (Koutsaki et al. 2011), and its decreased expression has been proposed to induce dyslipidemia and hyperinsulinemia (García-Fernández et al. 2008). Administration of exogenous IGF-1 leads to significantly reduced cholesterol levels in old mice relative to untreated old mice; however, cholesterol levels in treated mice still do not reach levels as low as those found in young, untreated mice (GarcíaFernández et al. 2008). Similar to uterine-ligated offspring, MPR offspring exhibit significantly reduced levels of Igf-1 at PND 21 and 130 (Fig. 4; Sohi et al. 2015). These offspring consequentially have reduced growth rate in comparison to control offspring, as indicated by a significantly lower body weight at PND 21 and PND 130 (Fig. 2). Given that this group of offspring also exhibits dyslipidemia in adult life (Sohi et al. 2011), it seems feasible that low levels of Igf-1 contribute to abnormally high levels of cholesterol. It is noteworthy that offspring exposed to a LP diet exclusively during lactation exhibit an even greater reduction in expression of hepatic Igf-1 (Sohi et al. 2015), which suggests that the neonatal window of development plays a significant role in the regulation of $I g f-1$ expression.

\section{Long-Term Outcomes III: Premature Aging}

Many studies have determined that there is an existing relationship between birth weight and longevity, and this is again due to alterations in fetal programming that underlie impeded fetal development. Lifespan becomes reduced when impaired fetal development is followed by postnatal catch-up growth, as demonstrated by studies 
a

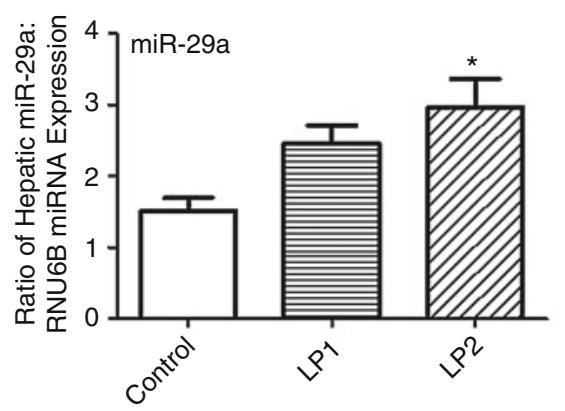

C

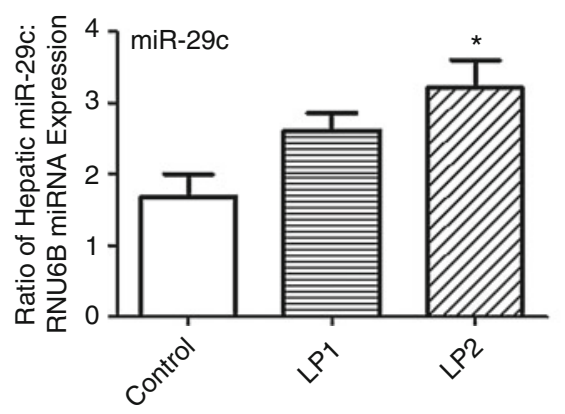

b

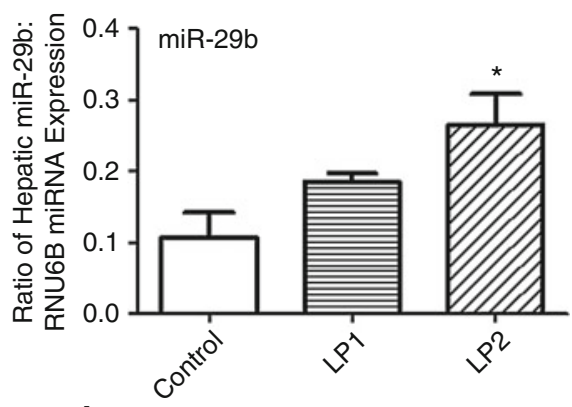

d

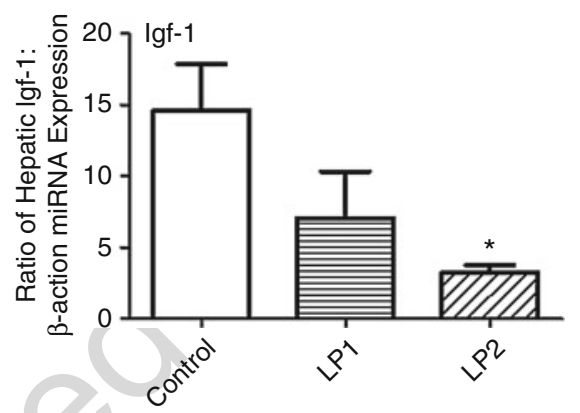

* $\mathrm{p}<0.05$ relative to Control

Fig. 4 Quantitative RT-PCR microRNA analysis of (a) miR-29a, (b) miR-29b, (c) miR-29c, and (d) Igf1 mRNA in the livers of rat offspring (Control, LP1, and LP2) derived at postnatal d130. Pregnant rats were given either a control diet (20\% protein) or a low protein diet ( $8 \%$ protein) during gestation only (LP1) and lactation (LP2). The relative amounts of miR-29a, 29b, and 29c mRNA were normalized to that the expression of RNU6B. The relative expression of each Igf1 mRNA transcript was normalized to that of the each b-actin mRNA transcript. Results were expressed as the mean \pm SEM. The groups were compared by ANOVA and significant difference was determined by a Tukey HSD post hoc test for individual pairwise comparisons $\left({ }^{*} P<0.05\right.$, indicates significance between control and LP2 cohort). For Fig. 2d, given the variances were not equal, the Tukey HSD post hoc test was performed on log-transformed data. $n=5-8$ /group, where each $n$ represents an offspring derived from a different mother (Reprinted from "Higher Hepatic MiR-29 Expression in Undernourished Male Rats During the Postnatal Period Targets the Long-term Repression of Insulin-like Growth Factor 1," G Sohi et al., Endocrinology (2015) 156(9): 3069-3076, with permission from Oxford University Press)

of the MPR diet by Ozanne and Hales (2004). Specifically, they demonstrated that MPR offspring have reduced fetal growth and these offspring tend to have increased lifespan when maintained on a LP diet (Hales et al. 1996). Conversely, MPR offspring that undergo postnatal catch-up growth after birth have a significantly reduced lifespan relative to their LP counterparts (16.3 vs. 13.1 months; Hales et al. 1996). Additionally, expression of sirtuin 1 (SIRT1) protein, a deacetylase enzyme believed to play a role in regulation of lifespan and glucose homeostasis (Michan and Sinclair 2007), was significantly decreased in skeletal muscle of MPR animals 
with postnatal catch-up growth (Chen et al. 2009). These offspring also have significantly decreased levels of insulin signaling molecules such as IGF-1 and phosphorylated IRS-1, so the authors predicted that impairments to insulin sensitivity may contribute to regulation of lifespan (Chen et al. 2009). A similar model of MPR also demonstrated that markers of cell senescence (e.g., p21 and p16) are significantly upregulated in pancreatic islets of recuperated rat offspring, as well as significantly shorter telomere length (Tarry-Adkins et al. 2009). This further consolidates the relationship between glucose homeostasis and longevity, given the role of pancreatic islets in insulin and glucagon production. It also is noteworthy that offspring born to normal protein mothers and cross-fostered to LP-fed dams during lactation (i.e., MPR lactation only) have significantly increased lifespan in comparison to control offspring (17.0 vs. 15.1 months; Hales et al. 1996). The authors speculated that offspring might therefore benefit from slow postnatal growth; however, adequate dietary protein still remains essential during pregnancy (Hales et al. 1996).

\section{Epigenetic Mechanisms Linking Protein Restriction and Adverse Metabolic Outcomes}

It is well understood that transcriptional changes directly compromise fetal development in utero; however the role of epigenetic alterations in fetal metabolic programming has not been investigated to great extent. Epigenetic mechanisms act to influence long-term gene expression without altering the primary genetic sequence, often by modifying interactions between transcriptional and/or translational machinery with regulatory sequences. Mechanisms such as direct DNA methylation, posttranslational histone modifications, and microRNAs (miRs) have been implicated in cases of fetal undernutrition, and LP-born offspring are no exception. In 2005, a study by Lillycrop et al. demonstrated that $\mathrm{CpG}$ island methylation status of hepatic glucocorticoid receptor $(G R)$ and PPAR $\alpha$ promoters are significantly reduced in MPR offspring, and this hypomethylated state is associated with increased expression of these genes. Interestingly, feeding of a LP diet in combination with folic acid supplementation prevented these epigenetic changes, indicating that one-carbon metabolism is essential in preventing the effects of this maternal insult (Lillycrop et al. 2005). Further studies also confirmed that this alteration exemplifies transgenerational effects, as methylation status is decreased in the F2 generation at PND 80 (Burdge et al. 2007). This is characteristic of many epigenetic mechanisms, thereby illustrating relevance of perinatal insult to health outcomes of future generations.

Chromatic structure is also greatly affected by posttranslational histone modifications, including histone acetylation, methylation, ubiquitination, ADP-ribosylation, and phosphorylation. In MPR, the long-term expression of gluconeogenic enzymes (e.g., G6Pase and 11 $\beta$-HSD1) is increased due to the histone-mediated silencing of hepatic liver X receptor alpha (LXR $\alpha$ ) at 4 months (Vo et al. 2013). LXR $\alpha$ is a transcription factor involved in the silencing of genes associated with glucose production. Vo et al. (2013) demonstrated that there is a significant decrease in histone $\mathrm{H} 3$ acetylation $[\mathrm{K} 9,14]$ at the transcriptional start site of $L x r \alpha$ in 4-month protein recuperated MPR 
offspring. This is concomitant with decreased association of LXR $\alpha$ at the LXR response element (LXRE) of G6Pase and 11 $\beta$-HSD1, culminating in glucose intolerance (Vo et al. 2013). As mentioned previously, MPR offspring also exhibit decreased expression of hepatic Cyp7a1 leading to hypercholesterolemia in male offspring at PND 21 and 130 (Sohi et al. 2011). This reduction in enzyme expression is due to epigenetic silencing at the Cyp $7 a 1$ promoter region, as there is increased tri-methylation and decreased acetylation of histone $\mathrm{H} 3[\mathrm{~K} 9,14]$, markers of chromatin condensation. It is interesting that female MPR offspring from the same cohort are protected from these histone modifications in adulthood, as they show complete opposite trends in methylation and acetylation.

In addition to DNA methylation and histone modifications, miRs have also been demonstrated to influence long-term gene expression via epigenetic mechanisms. MiRs are short, noncoding RNA molecules that act to silence target genes via target mRNA degradation or translational repression. In 2016, Su et al. investigated the role of miR-15b in pancreatic beta cell proliferation of MPR-born mouse offspring. It was discovered that miR-15b is significantly increased in the pancreatic islets of MPR offspring, accompanied by reduced expression of cyclin D1 and D2 (Su et al. 2016). Given the role of cyclins in progression through the cell cycle, it is believed that the downregulation of these molecules contributes to impaired beta cell function and thus glucose intolerance. As discussed earlier, administration of the MPR diet during pregnancy and lactation has been demonstrated to cause the upregulation of hepatic miR-29 expression in LP offspring with postnatal catch-up growth (Sohi et al. 2015). Each of miR-29a, miR-29b, and miR-29c were significantly increased in livers of 3 week and 4 month old offspring, and this further caused a reduction in expression of Igf-1 (Fig. 4; Sohi et al. 2015). With that in mind, it is possible that timing of nutritional restoration for IUGR offspring may play a role in long-term disease via modulation of miRs. Given that miRs also circulate in the blood, these animal studies could lead to novel therapeutic interventions with the use of miR inhibitors in neonatal treatment of the metabolic syndrome. An overview of the molecular mechanisms underlying MPR-induced metabolic dysfunction is illustrated in Fig. 5.

\section{Other Mechanisms Linking Protein Restriction and Adverse Metabolic Outcomes}

As previously mentioned, the fetal liver is proportionally small in MPR offspring at birth and undergoes rapid postnatal catch-up growth with introduction of a normal protein diet (Sohi et al. 2011; Hales et al. 1996). During this period of growth, hepatocytes undergo rapid replication such that the neonatal liver becomes larger. It is therefore possible that ER stress may contribute to poor metabolic health outcomes in the recuperated adult MPR offspring. ER stress is a cellular event which ensues due to environmental insults leading to an increase in the presence of misfolded or unfolded proteins present within the ER (Sohi et al. 2013). In response to ER stress, the unfolded protein response (UPR) becomes activated in attempt to reverse this 


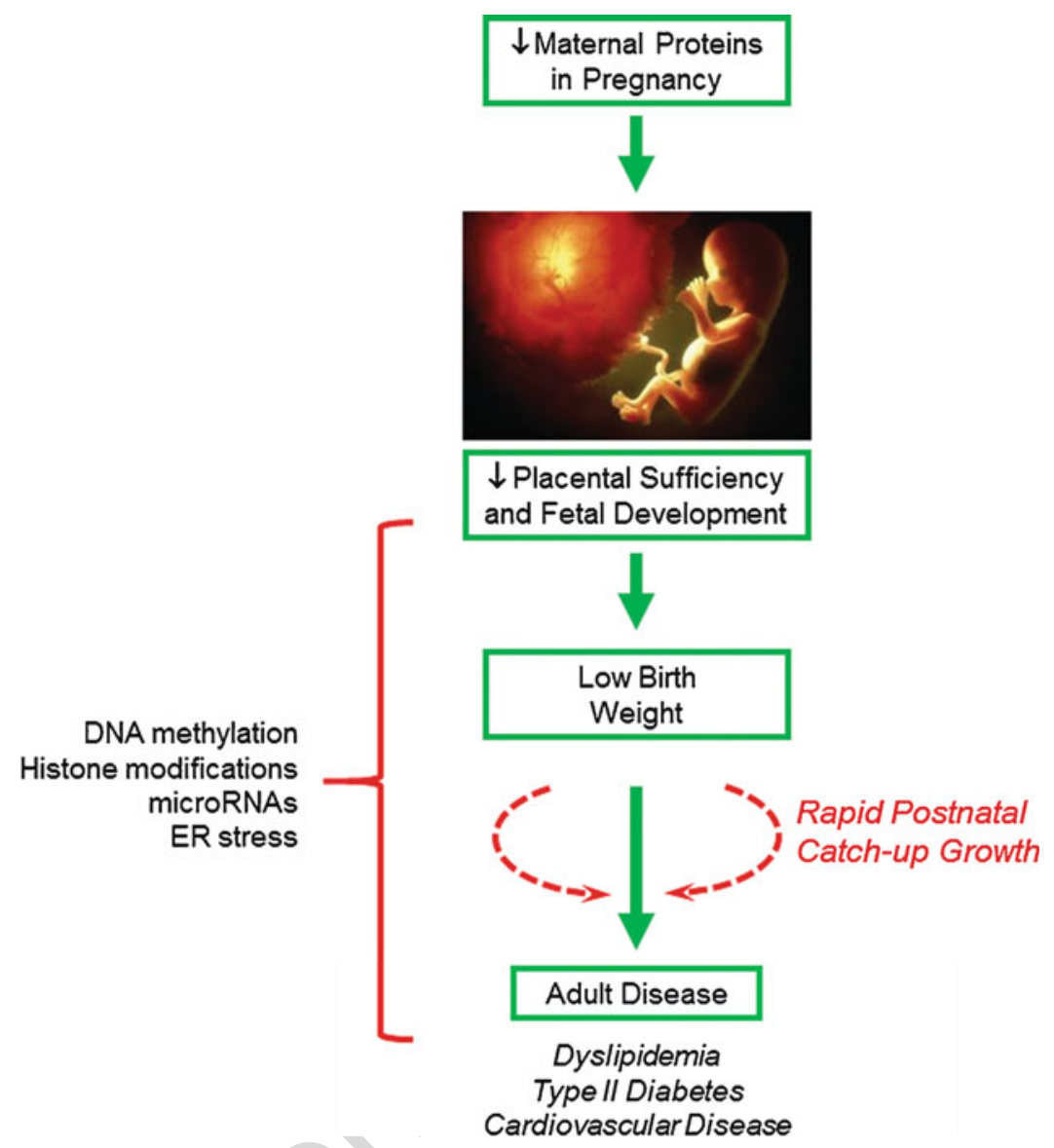

Fig. 5 Overview of the molecular mechanisms underlying how maternal protein restriction (MPR) during perinatal leads to long-term metabolic dysfunction in adulthood Direct pathways altered by maternal protein restriction are indicated by green solid arrows, while direct and indirect molecular mechanisms are indicated by red arrow

stress by refolding those misfolded proteins and/or attenuating protein translation through three signaling pathways (Sohi et al. 2013). In the case that the UPR cannot alleviate ER stress, apoptosis may further occur. The MPR offspring with catch-up growth undergo exhibit hepatic ER stress at 4 months of age as indicated by increased hepatic p-eIF2 $\alpha$ [Ser51], Grp78, and spliced Xbp1 (Sohi et al. 2013). P-eIF2 $\alpha$ [Ser51] is known to negatively regulate the initiation stage of protein translation (Proud 2005). As with many other mechanisms discussed in this review, the LP diet itself does not a play a direct role given embryonic day 19 low protein fetuses do not exhibit ER stress (Sohi et al. 2013). In addition to affecting hepatic function directly, ER stress may also be involved in the regulation of epigenetic mechanisms such as miRs. ER stress has been shown to cause an increase in 
miR-29a expression in a mouse model of amyotrophic lateral sclerosis (ALS; Nolan et al. 2014), and studies by Sohi et al. (2015) have implicated that MPR offspring with postnatal catch-up growth exhibit increased hepatic miR-29a and ER stress at 4 months of age. Considering that miR-29 targets Igf- 1 , this further suggests that ER stress may play an important role in the etiology of the metabolic syndrome in these IUGR offspring.

\section{Conclusion}

While maternal malnutrition exists in many forms, MPR has been shown to have major consequences for the long-term metabolic health of LP-exposed offspring. Epidemiological studies in humans have deduced that perinatal protein deficiency gives rise to low birth weight, and these individuals are at greater risk for development of the metabolic syndrome in adult life. Studies of individuals with SAM reveal that poor dietary protein can lead to glucose intolerance and abnormal plasma fatty acid levels. Moreover, animal studies of the MPR model have further established that LP-exposed offspring have low birth weight and asymmetrical IUGR, with liver growth and development taking a major hit relative to other organs. Additionally, the function of other organs such as the pancreas, muscle, and adipose becomes impaired, which further contributes to metabolic dysfunction. In adult life, these animals tend to have glucose intolerance, dyslipidemia, and increased visceral obesity. Onset of these deficits are further exacerbated by postnatal catch-up growth, as a nutrient-poor prenatal environment gives rise to altered fetal programming that is not beneficial in a nutrient-rich postnatal environment. Furthermore, offspring with postnatal catch-up growth exhibit reduced lifespan relative to animals that are fed either a control or LP-exclusive diet. While the mechanisms behind these defects are not fully understood, it is widely accepted that epigenetic alterations such as DNA methylation, posttranslational histone modifications, and miRs can influence fetal gene expression. Animal models of MPR give us great insight into what might be occurring in humans, and so further investigation is required to better comprehend the molecular basis of the metabolic syndrome in response to perinatal protein restriction. Until then, nutritional intervention during pregnancy is necessary to ensure that mothers consume appropriate amounts of dietary protein such that there are no negative effects to fetal growth and development.

\section{Policies and Protocols}

In this review, we have discussed the metabolic implications of perinatal protein restriction and postnatal catch-up growth in LP-born offspring. Models of protein restriction have confirmed that insufficient amino acids during pregnancy contribute to low birth weight, and this leads to the metabolic syndrome in adult life. Due to fetal adaptations that occur in utero, low birth weight offspring have rapid weight gain when presented with a mismatched postnatal environment (i.e., a "normal" 
protein diet), exacerbating the risk for adult metabolic disease. It is critical that primary health-care workers are informed regarding this information related to postnatal catch-up growth. Physicians, nurses, and midwives should emphasize to patients that a balance between prenatal and postnatal diet with respect to protein intake is essential. In addition, it is recommended that pregnant women ingest protein in the form of animal-sourced amino acids rather than plant-based amino acids. As mentioned, studies have shown that poor fetal pancreatic development (due to perinatal protein restriction) can be rescued with administration of taurine, a meat-sourced amino acid (Boujendar et al. 2003). While the role of animal-based amino acids has been only investigated in pancreatic development, it is conceivable that this may be the case for other metabolic organs as well. As always, prevention is a more successful strategy than treatment; therefore, it is highly encouraged that health-care workers and pregnant mothers work together to prevent maternal malnutrition for the sake of the developing fetus.

\section{Dictionary of Terms}

Asymmetrical intrauterine growth restriction (IUGR)

Dyslipidemia

Epigenetics

Endoplasmic reticulum stress

Gluconeogenesis

Glucose intolerance

Heterochromatin

Malnutrition
A category of IUGR in which infants are not only small for gestational age but also exhibit disproportionately small organ size.

An increase in plasma cholesterol, triglycerides, or both, leading to the development of cardiovascular disease.

The study of heritable changes in gene expression without modification of the primary gene sequence.

A cellular stress response characterized by increased accumulation of misfolded and/or unfolded polypeptides in the lumen of the endoplasmic reticulum.

Production of de novo glucose molecules from noncarbohydrate sources.

A prediabetic condition in which affected individuals exhibit elevated blood glucose (i.e., hyperglycemia) in the fasted and/or fed state. Glucose intolerance often precedes type II diabetes, which occurs when individuals also exhibit insulin resistance.

Repressed region of DNA leading to a decrease in gene expression.

Either an excess or deficiency in one or more nutrients. 
Metabolic syndrome

MicroRNAs

Placental insufficiency

Postnatal catch-up growth

Senescence

Severe acute malnutrition (SAM)

Telomere
A group of adverse metabolic symptoms that together confer increased risk for type II diabetes mellitus and cardiovascular disease.

Endogenous, short, noncoding RNA molecules that posttranscriptionally regulate expression of target mRNA sequences.

An idiopathic condition occurring in $8 \%$ of pregnancies that leads to reduced maternofetal nutrient exchange due to inadequate placental blood flow.

A period of growth after birth whereby low birth weight offspring exhibit rapid growth rate such that they "catch-up" to average body weight. Offspring that undergo postnatal catch-up growth are often referred to as "recuperated" offspring.

The process of biological aging due to loss of cellular division and function.

An extreme form of undernutrition characterized by muscle atrophy and low body weight can be further categorized into cases of marasmus (extreme caloric restriction) or kwashiorkor (extreme protein deficiency).

A protective region of repetitive sequences at the end of a chromosome.

\section{Summary Points}

- In mammals, many organs are vulnerable to perinatal protein deficiency, which causes altered gene expression and leads to long-term metabolic effects in the offspring.

- Rat offspring exposed to maternal protein restriction have low birth weight and asymmetrical intrauterine growth restriction (i.e., many organs are proportionally small relative to the rest of the body).

- Given the role of the liver in glucose homeostasis, as well as the metabolism of cholesterol and a variety of drugs, impaired liver growth and development by maternal protein restriction leads to abnormal regulation of plasma glucose levels and hepatic enzymes.

- Adipose tissue plays an important role in lipid storage and insulin signaling. 
- Altered hepatic, pancreatic, or adipose function leads to dyslipidemia, obesity, glucose intolerance, and coronary artery disease.

- Transcriptional and epigenetic mechanisms (e.g., DNA methylation, posttranslational histone modifications, microRNAs) facilitate adaptation of developing organs to amino acid deficiencies in utero; however, this can have dire consequences long-term and may have transgenerational effects.

- Endoplasmic reticulum stress is present in offspring with postnatal catch-up growth due to rapid growth of metabolic organs, and activation of the UPR further increases risk for the metabolic syndrome in adult life.

Acknowledgements Canadian Institutes for Health Research Operating Grant and Natural Sciences and Engineering Research Council of Canada Operating Grant.

\section{References}

Badaloo AV, Forrester T, Reid M, Jahoor F (2006) Lipid kinetic differences between children with kwashiorkor and those with marasmus. Am J Clin Nutr 83:1283-1288

Barker DJ (1994) Outcome of low birthweight. Horm Res 42:223-230

Barker D (2003) The midwife, the coincidence, and the hypothesis. BMJ 327:1428-1430

Barker DJ, Martyn CN, Osmond C, Hales CN, Fall CH (1993) Growth in utero and serum cholesterol concentrations in adult life. BMJ 307:1524-1527

Barker DJ, Eriksson JG, Forsen T, Osmond C (2002) Fetal origins of adult disease: strength of effects and biological basis. Int J Epidemiol 31:1235-1239

Bieswal F, Ahn MT, Reusens B, Holvoet P, Raes M, Rees WD, Remacle C (2006) The importance of catch-up growth after early malnutrition for the programming of obesity in male rat. Obesity Silver Spring 14:1330-1343

Blesson CS, Chinnathambi V, Kumar S, Yallampalli C (2017) Gestational protein restriction impairs glucose disposal in the gastrocnemius muscles of female rats. Endocrinology 158(4):756-767

Bol VV, Delattre A-I, Reusens B, Raes M, Remacle C (2009) Forced catch-up growth after fetal protein restriction alters the adipose tissue gene expression program leading to obesity in adult mice. Am J Physiol Regul Integr Comp Physiol 297:R291-R299

Boujendar S, Reusens B, Merezak S, Ahn MT, Arany E, Hill D, Remacle C (2002) Taurine supplementation to a low protein diet during foetal and early postnatal life restores a normal proliferation and apoptosis of rat pancreatic islets. Diabetologia 45:856-866

Boujendar S, Arany E, Hill D, Remacle C, Reusens B (2003) Taurine supplementation of a low protein diet fed to rat dams normalizes the vascularization of the fetal endocrine pancreas. J Nutr $133: 2820-2825$

Burdge GC, Slater-Jefferies J, Torrens C, Phillips ES, Hanson MA, Lillycrop KA (2007) Dietary protein restriction of pregnant rats in the $\mathrm{F} 0$ generation induces altered methylation of hepatic gene promoters in the adult male offspring in the F1 and F2 generations. Br J Nutr 97:435-439

Burns SP, Desai M, Cohen RD, Hales CN, Iles RA, Germain JP, Going TC, Bailey RA (1997) Gluconeogenesis, glucose handling, and structural changes in livers of the adult offspring of rats partially deprived of protein during pregnancy and lactation. J Clin Invest 100:1768-1774

Chamson-Reig A, Thyssen SM, Arany E, Hill DJ (2006) Altered pancreatic morphology in the offspring of pregnant rats given reduced dietary protein is time and gender specific. J Endocrinol 191:83-92

Chamson-Reig A, Thyssen SM, Hill DJ, Arany E (2009) Exposure of the pregnant rat to low protein diet causes impaired glucose homeostasis in the young adult offspring by different mechanisms in males and females. Exp Biol Med Maywood 234:1425-1436 
Chen J-H, Martin-Gronert MS, Tarry-Adkins J, Ozanne SE (2009) Maternal protein restriction affects postnatal growth and the expression of key proteins involved in lifespan regulation in mice. PLoS One 4:e4950

Craig WJ, Mangels AR (2009) Position of the American Dietetic Association: vegetarian diets. J Am Diet Assoc 109:1266-1282

Crosby WM (1991) Studies in fetal malnutrition. Am J Dis Child 1960(145):871-876

Desai M, Hales CN (1997) Role of fetal and infant growth in programming metabolism in later life. Biol Rev Camb Philos Soc 72:329-348

Eriksson JG (2006) Early growth, and coronary heart disease and type 2 diabetes: experiences from the Helsinki Birth Cohort Studies. Int J Obes 2005 30(Suppl 4):S18-S22

Ferreira DJS, da Silva Pedroza AA, Braz GRF, da Silva-Filho RC, Lima TA, Fernandes MP, Doi SQ, Lagranha CJ (2016) Mitochondrial bioenergetics and oxidative status disruption in brainstem of weaned rats: immediate response to maternal protein restriction. Brain Res 1642:553-561

Forrester TE, Badaloo AV, Boyne MS, Osmond C, Thompson D, Green C, Taylor-Bryan C, Barnett A, Soares-Wynter S, Hanson MA et al (2012) Prenatal factors contribute to the emergence of kwashiorkor or marasmus in severe undernutrition: evidence for the predictive adaptation model. PLoS One 7:e35907

Francis-Emmanuel PM, Thompson DS, Barnett AT, Osmond C, Byrne CD, Hanson MA, Gluckman PD, Forrester TE, Boyne MS (2014) Glucose metabolism in adult survivors of severe acute malnutrition. J Clin Endocrinol Metab 99:2233-2240

García-Fernández M, Delgado G, Puche JE, González-Barón S, Castilla Cortázar I (2008) Low doses of insulin-like growth factor I improve insulin resistance, lipid metabolism, and oxidative damage in aging rats. Endocrinology 149:2433-2442

Guan H, Arany E, van Beek JP, Chamson-Reig A, Thyssen S, Hill DJ, Yang K (2005) Adipose tissue gene expression profiling reveals distinct molecular pathways that define visceral adiposity in offspring of maternal protein-restricted rats. Am J Physiol Metab 288:E663-E673

Hadden DR (1967) Glucose, free fatty acid, and insulin interrelations in kwashiorkor and marasmus. Lancet 290:589-593

Hales CN, Barker DJ (2001) The thrifty phenotype hypothesis. Br Med Bull 60:5-20

Hales CN, Barker DJ, Clark PM, Cox LJ, Fall C, Osmond C, Winter PD (1991) Fetal and infant growth and impaired glucose tolerance at age 64. BMJ 303:1019-1022

Hales CN, Desai M, Ozanne SE, Crowther NJ (1996) Fishing in the stream of diabetes: from measuring insulin to the control of fetal organogenesis. Biochem Soc Trans 24:341-350

Honório de Melo Martimiano P, de Sa Braga Oliveira A, Ferchaud-Roucher V, Croyal M, Aguesse A, Grit I, Ouguerram K, Lopes de Souza S, Kaeffer B, Bolaños-Jiménez F (2017) Maternal protein restriction during gestation and lactation in the rat results in increased brain levels of kynurenine and kynurenic acid in their adult offspring. J Neurochem 140:68-81

Koutsaki M, Sifakis S, Zaravinos A, Koutroulakis D, Koukoura O, Spandidos DA (2011) Decreased placental expression of hPGH, IGF-I and IGFBP-1 in pregnancies complicated by fetal growth restriction. Growth Horm IGF Res 21:31-36

Lillycrop KA, Phillips ES, Jackson AA, Hanson MA, Burdge GC (2005) Dietary protein restriction of pregnant rats induces and folic acid supplementation prevents epigenetic modification of hepatic gene expression in the offspring. J Nutr 135:1382-1386

Mariani A, Chalies S, Jeziorski E, Ludwig C, Lalande M, Rodière M (2009) Consequences of exclusive breast-feeding in vegan mother newborn - case report. Arch Pediatr 16:1461-1463

Michan S, Sinclair D (2007) Sirtuins in mammals: insights into their biological function. Biochem J 404:1-13

Nolan K, Mitchem MR, Jimenez-Mateos EM, Henshall DC, Concannon CG, Prehn JH (2014) Increased expression of microRNA-29a in ALS mice: functional analysis of its inhibition. J Mol Neurosci 53:231-241

Ogata ES, Bussey ME, Finley S (1986) Altered gas exchange, limited glucose and branched chain amino acids, and hypoinsulinism retard fetal growth in the rat. Metabolism 35:970-977 
de Oliveira JC, Gomes RM, Miranda RA, Barella LF, Malta A, Martins IP, Franco CC da S, Pavanello A, Torrezan R, Natali MRM et al (2016) Protein restriction during the last third of pregnancy malprograms the neuroendocrine axes to induce metabolic syndrome in adult male rat offspring. Endocrinology 157:1799-1812

Ong KK, Ahmed ML, Emmett PM, Preece MA, Dunger DB (2000) Association between postnatal catch-up growth and obesity in childhood: prospective cohort study. BMJ 320:967-971

Ozanne SE, Hales CN (2004) Lifespan: catch-up growth and obesity in male mice. Nature 427:411-412

Ozanne SE, Wang CL, Coleman N, Smith GD (1996a) Altered muscle insulin sensitivity in the male offspring of protein-malnourished rats. Am J Phys 271:E1128-E1134

Ozanne SE, Smith GD, Tikerpae J, Hales CN (1996b) Altered regulation of hepatic glucose output in the male offspring of protein-malnourished rat dams. Am J Phys 270:E559-E564

Petrik J, Reusens B, Arany E, Remacle C, Coelho C, Hoet JJ, Hill DJ (1999) A low protein diet alters the balance of islet cell replication and apoptosis in the fetal and neonatal rat and is associated with a reduced pancreatic expression of insulin-like growth factor-II. Endocrinology 140:4861-4873

Petry CJ, Ozanne SE, Hales CN (2001) Programming of intermediary metabolism. Mol Cell Endocrinol 185:81-91

Phipps K, Barker DJ, Hales CN, Fall CH, Osmond C, Clark PM (1993) Fetal growth and impaired glucose tolerance in men and women. Diabetologia 36:225-228

Piccoli GB, Clari R, Vigotti FN, Leone F, Attini R, Cabiddu G, Mauro G, Castelluccia N, Colombi N, Capizzi I et al (2015) Vegan-vegetarian diets in pregnancy: danger or panacea? A systematic narrative review. BJOG 122:623-633

Proud CG (2005) eIF2 and the control of cell physiology. Semin Cell Dev Biol 16:3-12

Ravelli GP, Stein ZA, Susser MW (1976) Obesity in young men after famine exposure in utero and early infancy. N Engl J Med 295:349-353

Ravelli AC, van der Meulen JH, Michels RP, Osmond C, Barker DJ, Hales CN, Bleker OP (1998) Glucose tolerance in adults after prenatal exposure to famine. Lancet 351:173-177

Simmons RA, Gounis AS, Bangalore SA, Ogata ES (1992) Intrauterine growth retardation: fetal glucose transport is diminished in lung but spared in brain. Pediatr Res 31:59-63

Sohi G, Weese K, Revesz A, Arany E, Hardy DB (2011) Maternal protein restriction elevates cholesterol in adult rat offspring due to repressive changes in histone modifications at the cholesterol 7 $\alpha$-hydroxylase promoter. Mol Endocrinol 25(5):785-798

Sohi G, Revesz A, Hardy DB (2013) Nutritional mismatch in postnatal life of low birth weight rat offspring leads to increased phosphorylation of hepatic eukaryotic initiation factor $2 \alpha$ in adulthood. Metabolism 62(10):1367-1374

Sohi G, Barry E, Velenosi TJ, Urquhart BL, Hardy DB (2014) Nutritional mismatch in postnatal life of low birth weight rat offspring leads to elevated hepatic Сyp3a and Cyp2c activity in adulthood. Drug Metab Dispos 42:221-228

Sohi G, Revesz A, Ramkumar J, Hardy DB (2015) Higher hepatic miR-29 expression in undernourished male rats during the postnatal period targets the long-term repression of IGF-1. Endocrinology 156:3069-3076

Spoelstra MN, Mari A, Mendel M, Senga E, van Rheenen P, van Dijk TH, Reijngoud D-J, Zegers RGT, Heikens GT, Bandsma RHJ (2012) Kwashiorkor and marasmus are both associated with impaired glucose clearance related to pancreatic $\beta$-cell dysfunction. Metabolism 61:1224-1230

Su Y, Jiang X, Li Y, Li F, Cheng Y, Peng Y, Song D, Hong J, Ning G, Cao Y et al (2016) Maternal low protein isocaloric diet suppresses pancreatic $\beta$-cell proliferation in mouse offspring via miR-15b. Endocrinology 157:4782-4793

Tarry-Adkins JL, Chen JH, Smith NS, Jones RH, Cherif H, Ozanne SE (2009) Poor maternal nutrition followed by accelerated postnatal growth leads to telomere shortening and increased markers of cell senescence in rat islets. FASEB J 23:1521-1528 
Vo T, Revesz A, Ma N, Hardy DB (2013) Maternal protein restriction leads to enhanced hepatic gluconeogenic gene expression in adult male rat offspring due to impaired expression of the liver x receptor. J Endocrinol 218:85-97

Williams SJ, Campbell ME, McMillen IC, Davidge ST (2005) Differential effects of maternal hypoxia or nutrient restriction on carotid and femoral vascular function in neonatal rats. Am J Physiol Integr Comp Physiol 288:R360-R367

Zhang T, Guan H, Arany E, Hill DJ, Yang K (2007) Maternal protein restriction permanently programs adipocyte growth and development in adult male rat offspring. $\mathrm{J}$ Cell Biochem 101:381-388

Zheng S, Rollet M, Pan Y-X (2012) Protein restriction during gestation alters histone modifications at the glucose transporter 4 (GLUT4) promoter region and induces GLUT4 expression in skeletal muscle of female rat offspring. J Nutr Biochem 23:1064-1071 